\title{
REAL-TIME LABEL INSERTION IN LIVE VIDEO THROUGH ONLINE TRIFOCAL TENSOR ESTIMATION
}

\author{
Robert Laganière \\ School of Information Technology and Engineering \\ University of Ottawa \\ Email:laganier@site.uottawa.ca \\ Johan Gottin \\ École Supérieure d'Ingénieurs d'Annecy \\ Annecy, FRANCE \\ Email: johan.gottin@esia.fr
}

\begin{abstract}
Keywords: Augmented reality, trifocal tensor transfer, automatic labelling.
Abstract: We present an augmented reality application that can supplement a live video sequence with virtual labels associated with the scene content captured by an agile video camera moving inside an explored environment. The method proposed is composed of two main phases. First, a matching phase where reference images are successively compared with the captured images. And, second, a tracking phase that aims at maintaining the correspondence between a successfully matched reference image and each frame of a captured sequence. Labels insertion is based on projective transfer using the trifocal tensor, this one being estimated and continuously updated as the camera is moved inside the scene.
\end{abstract}

\section{INTRODUCTION}

Augmented reality (AR) is the technique of choice when one wants to supplement a user's view with useful information concerning an observed scene. Among the many ways by which content aware information can be added to a video sequence, adding text annotations is probably one of the simplest but effective way to proceed. Under this mode, some of the scene objects are associated with informative texts that are overlaid on the video when these objects become visible. The displayed information often consists in text labels pointing to the object's parts they describe. Such system would be particulary useful to a mobile user exploring an unfamiliar environment. Using a simple PDA equipped with a camera, or a more sophisticated HMD, virtual overlays would appear on the images collected by the device, helping the user in understanding the scene content. A possible use scenario is the case of a tourist visiting an art gallery and for whom the supplemented labels would provide information about the exhibited objects. Alternatively, the system could assist a technician in the inspection of a given building; the added virtual labels would then represent a source of complementary information (such as inventory numbers, ownership, etc.) to help him in the accomplishment his tasks.

The augmented reality application presented here is an automatic annotation system that can insert, at frame rate, virtual labels associated with the scene content captured by an agile video camera that freely moves inside an explored environment. The core of this application relies on an online-tensor estimation scheme that has been presented in ( $\mathrm{Li}$ et al., 2004) and that has proven to be effective in AR. In this latter application, virtual objects are added to a scene by transferring a virtual marker from two reference views to the current view. We use here the same concept to transfer labels from reference views to a video frame with the difference that multiple reference images are now used. Also, a matching phase has been introduced to detect when the current video is visualizing scene elements for which textual annotations are available and to identify the reference frames on which this information has been entered. The method proposed here is therefore composed of two phases. First, this matching phase where the reference images are successively compared with a current frame. And, second, a tracking phase where the correspondence between a successfully matched reference image and each frame of the captured sequence is maintained.

An important asset of the proposed method is that it doesn't require estimation of the camera pose, neither the use of some external localization sensor or the recourse to some special markers inserted, beforehand, inside the scene. Labels insertion is based on projective transfer using the trifocal tensor, this one being estimated and continuously updated as the camera is 
moved inside the scene. The estimation scheme we have developed demonstrates that it is possible to obtain quick and reliable estimates of the trifocal tensor. A simple tracker is used to provide an evolving set of point triplets. Stable performance of tracking over long video sequences is also ensured through the automatic recovering of lost points and the removal of wrong traces using trifocal transfer.

The next section briefly reviews some existing systems while Section 3 presents the trifocal tensor. Sections 4 and 5 describe the proposed online labelling procedure. Section 6 explains our online tensor estimation scheme. Results of video frame augmentation are presented in Section 7. Section 8 is a conclusion.

\section{RELATED WORKS}

Most of the existing automatic scene annotation systems rely on the use of positional sensors in order to determine user's 3D position and viewing direction. In addition, an accurate model, describing the current position of the objects of interest, is also required. This is the case of the system in (Newman et al., 2001) that uses ultrasonic pulses that are read by receivers located at fixed position in the ceiling. Accurate 3D position is then obtained from times-of-flight calculation. The objective of the augmented reality system in (Bell et al., 2002) is to provide a situation-awareness aid to a user in the form of a world in miniature representation of the environment and that is embedded in the user's view. By selecting specific objects, either in the scene image or in the virtual miniature representation, pop-up annotations are displayed.

General real-time AR systems can also be used for virtual label insertion. In the calibration-free system described in (Kutulakos and Vallino, 1998), four or more non-coplanar points are tracked along the video and an affine object representation is used to overlay virtual objects on a video stream. However, the used control points have to be visible in every frame, which restricts the range of views in which augmentations can take place. The method in (Lourakis and Argyos, 2004) is able to recover the camera positions in close to real-time through the chaining of homographies computed from the tracking of 3D planes. The AR system proposed in (Chia et al., 2002) computes camera pose by using the epipolar constraints that exists between every video frame and two keyframes. An alternative method consists in linking the video frames with two keyframes; trifocal tensor is then computed to transfer the location of the virtual object from the keyframes (Boufama and Habed, 2005). Another recent system that performs very well in real time scene augmentation is the one proposed in (Vacchetti et al., 2004). In addition to keyframes, the system also needs a 3D model of the target object. By matching the current frame with a preregistered keyframe, 2D-3D correspondences are obtained using which virtual augmentation can be performed.

These approaches generally necessitate full calibration information, including all camera projection matrices associated to the selected keyframes. AR systems not requiring any metric information would be more flexible and easier to deploy. This is the solution proposed by the application described here.

\section{THE TRIFOCAL TENSOR}

The augmented reality system proposed here relies on projective geometry concepts, well-known in computer vision, and that describes the relation between the multiple views of a same scene. In particular, the trifocal tensor which is a mathematical entity describing three-view geometry. It can represented by a $3 \times 3 \times 3$ matrix $\mathbf{T}$. This one can be computed from at least 5 correspondences over the three views. The key benefit brought by this tensor matrix resides in the fact that if a match $\left(\mathbf{x}, \mathbf{x}^{\prime}\right)$ is already known between the first two images, the position of the matching point $\mathrm{x}^{\prime \prime}$ in the third image can be determined exactly using:

$$
x_{l}^{\prime \prime}=x_{i}^{\prime} \sum_{k=1}^{3} x_{k} T_{k j l}-x_{j}^{\prime} \sum_{k=1}^{3} x_{k} T_{k i l}
$$

which defines 9 trilinearities for $i, j \in\{1,2,3\}, 4$ of which are linearly independent. In the context of automatic label insertion, this means that if the tensor relation between two reference images and a current view can be maintained, then the labels specified in these reference views can be transferred to the current frame at their appropriate locations. This is the strategy that is exploited here.

In the case of two views only, the projective relation is described by a $3 \times 3$ matrix, the fundamental matrix. This one defines an epipolar constraint that states that the homolog of any point will necessarily lies on a line (the epipolar line of that point) in the other view. One use of this constraint is to validate putative match pairs by verifying if the proposed matched point indeed lie on the corresponding epipolar lines. Note that the trifocal tensor can also be used to validate triplet of matches. Indeed, the trilinear constraint (1) is always satisfied for good matches.

Tensor estimation and point transfer are used by the online label insertion process. Guided matching and match validation based on fundamental and tensor matrices are used during the label specification phase as explained in the sections to follow. 


\section{LABEL SPECIFICATION}

The initial phase consists in specifying label locations on the reference images. In order to be able to transfer these labels on arbitrary views, it is required to specify each of them on at least two reference views. However, since the tensor estimation process requires a set of good matched points, three views of each object of interest are used as it is the most favorable configuration to obtain reliable feature matching.

The reference images are captured by moving a camera at three different locations. Points are detected in the first image and as the camera is moved from location 2 and 3 , these points are tracked from frame to frame (we used here the Lucas-Kanade tracker). The points successfully tracked from the first reference image to the third one constitute an initial match set that can then be validated and refined. To do so, we use an approach that combines the RANSAC projective procedure described in (Roth and Whitehead, 2000) and the calibrated matching procedure proposed in (Vincent and Laganière, 2002). The net result is therefore three reference images for which a rich and reliable set of matched triplets is available. This pool of matches will be used during the online label insertion procedure.

The interactive label insertion procedure can then be undertaken using these three reference images. Each label is inserted by first specifying its location in one reference image. Its location on a second reference image is then specified, but this time guided by the epipolar geometry (computed from the available match set), as shown in Figure 1. The location of the label in the third image does not have to be specified, as this one can be computed by virtue of the trifocal tensor transfer property.

Once all the labels, for each object of interest have been inserted, the live video augmentation application can be launched.

\section{ONLINE LABEL INSERTION}

When the application is running, images are continuously acquired using a camera that is freely moved inside the scene. The online label insertion procedure is composed of two phases: a matching phase, where references images are compared with the video frames in order to determine which labels have to be inserted, and a tracking phase that allows to keep displaying the labels at their appropriate locations. The complete process is described in Figure 2.
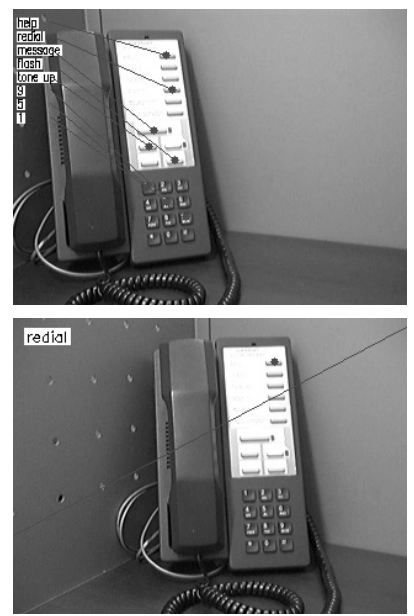

Figure 1: Specification of the virtual labels in reference image 1 (top) and guided insertion (i.e. the associated point must be on the epipolar line shown) of 'redial' label in the second reference image (bottom).

\subsection{The Matching Phase}

Ideally, each of the captured frame should be compared with all the stored reference images. However, even if feature-based matching is applied here, such an exhaustive matching procedure could be quite costly. For this reason, matching is performed on only a subset of the available reference images. At the next capture, a different subset is selected. This strategy allows keeping the processing rate sufficiently high while giving access to a large quantity of reference images. Obviously, it might take few captures before a successful match is obtained, but with an adequate frame rate, the response can remain acceptable.

Each putative matching between a video frame and a reference image is then validated by checking if the established match set is supported by a valid 2 -view geometry. In order to reduce the computational cost of this operation, an accelerated random sampling strategy is applied here. Eight feature matches are randomly selected and the corresponding fundamental matrix is computed. The match between the two images will be considered to be good, if a large number of individual matches supports this fundamental matrix; that is if for a given match pair, one point lies close to the epipolar line of the other point, as computed by this matrix. To speed up this validation, the test is performed using only 8 match pairs among which 6 must support the geometry in order for the match to be accepted. That simplified strategy does not fully guarantees the validity of a match, but the probability that a wrongly matched pair of images survives to this test is nevertheless very low. Once an image match pair has been accepted, an additional filter- 


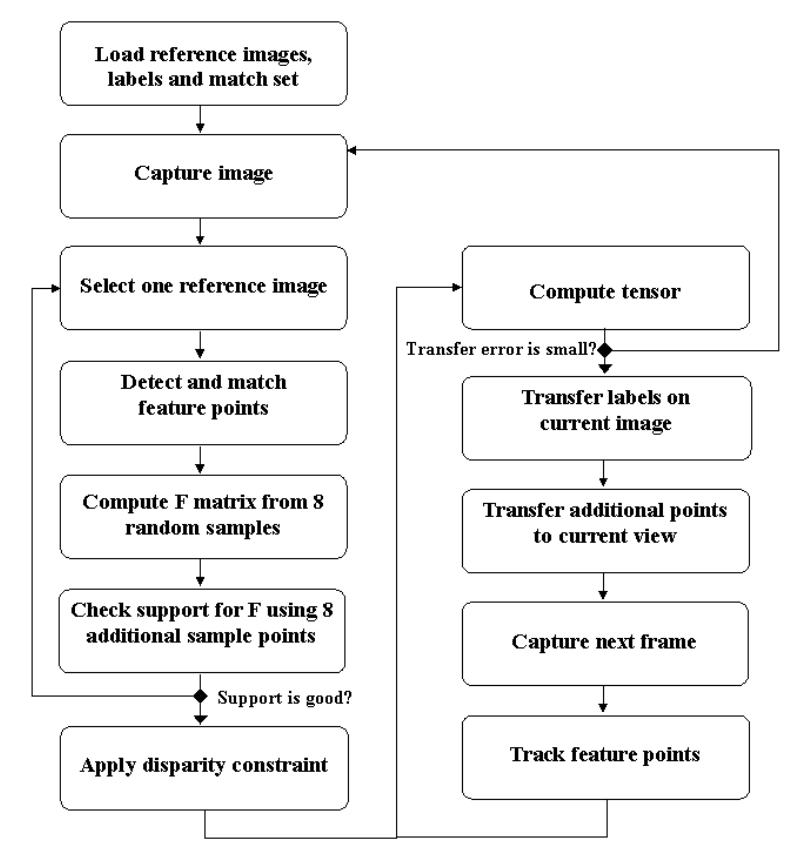

Figure 2: The virtual label insertion process.

ing step is applied on the proposed match set in order to eliminate the obvious mismatches that remain. The disparity constraint has proven to be quick and effective in performing this task (Vincent and Laganière, 2001). It consists in eliminating any feature match exhibiting a disparity vector that largely differs from its neighbors. At this stage, a match is considered to have been established between a reference image and the currently seen image. This latter can now be supplemented with the appropriate virtual labels; this is done at the tracking phase.

\subsection{The Tracking Phase}

As mentioned previously, the transfer of the labels from the reference image to the current is done using the trifocal tensor. This is possible because each reference image is associated with two other reference images, forming a triplet for which a rich match set is available (as explained in Section 4). This means that the image matching obtained is, in fact, a matching between one image and the three images of a series of reference images. It is therefore possible to compute a tensor between a current frame and two of the images of the series. This tensor must however be computed quickly in order to keep the frame rate high. This computation must also be robust, as some false matches are probably present in the match set found during the matching phase. In addition, the estimated tensor must have a good accuracy in order to produce well localized scene labels. For this reason, the the tensor is estimated using an algebraic minimization method and its accuracy is improved afterwards through a quick outlier removal step. The details of this online tensor estimation procedure are given in the next section.

With an accurate tensor in hands, it is easy to transfer the labels from the reference images to the current view. The points that have been matched in this current view are then tracked from frame to frame in order to maintain the relation with the current series of reference images. At each new camera position, the tensor must be re-estimated with the match set that includes the current frame points at their currently tracked location. It is important to note that, when points are tracked over time, more and more features are unavoidably lost. If nothing is done, the tracked set will eventually vanish. To overcome this problem, the match set is updated after each tensor estimation. Indeed, using the pool of matches available in the reference images, it is possible to transfer additional points on the image using that newly estimated tensor. This last step ensures the long term viability of the tracking phase.

\section{ONLINE ESTIMATION OF THE TENSOR}

In this section we describe a method to quickly estimate the trifocal tensor based on the use of two reference frames. This approach has been introduced in (Li et al., 2004) for augmenting a scene in realtime with virtual objects.

The trifocal tensor describes the projective geometric relation of image triplets taken from cameras. It can be conveniently estimated using the so-called Algebraic Minimization method (Hartley and Zisserman, 2000). However, in the present application, two problems have to be overcome: first, the tensor estimation process must be fast; and second, the estimated tensors must be accurate. This is to say that we have to use all available matches when estimating a tensor. Consequently, we have to counter the effect of false matches introduced in the matching phase and also, in the tracking phase. In fact, it is during this latter phase that outlier rejection is the most crucial. Indeed, it is unavoidable that the tracker will lose some features, and will introduce some wrong traces. This is especially true in the case of sequences produced by handheld cameras involving quick and saccadic motion. The estimation process therefore has to be robust to the presence of outliers. In order to solve this problem, we developed an estimation scheme that exploits the geometrical properties of the problem and that uses rapid robust estimation strategies.

One important properties of our system geometry 


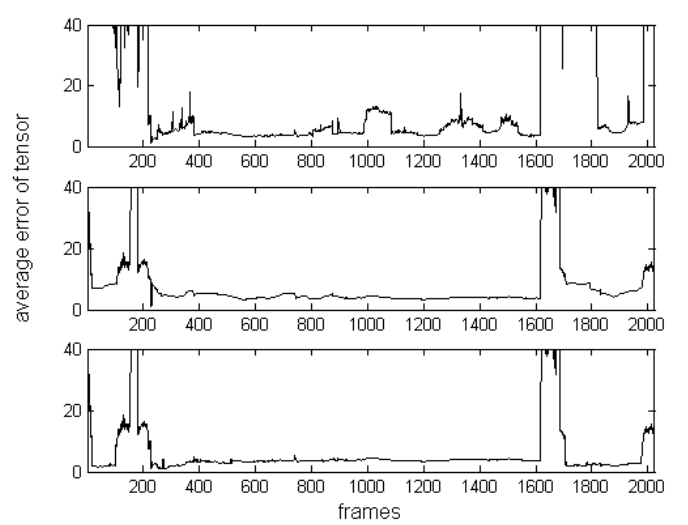

Figure 3: Average transfer error on the computed tensors in a sequence of 1749 frames. Top: the tensors computed from all putative triplets using AM; Middle: the improvements achieved by using fixed projection matrices; Bottom: the resulting tensors refined by applying the $\mathrm{x} 84$ rule.

resides in the fact that the tensors to be estimated are always associated with two fixed views (the reference frames). Since the algebraic method uses parametrization of the projection matrices, we therefore have two of the three projection matrices known which reduces the dimensionality of the problem. Our experimentations have also shown that tensor estimation subject to a fixed projection matrices exhibit reliable performance over long sequences. In addition, it has the capability to attenuate the effect of false matches and stabilizes the estimation results when only few features are being tracked.

Each time a new tensor is computed using the algebraic minimization approach, the average value of the residual errors is then computed in order to assess the quality of the resulting transfer. If its value is smaller than a given threshold (we used 3 pixels), then the tensor is judged to be of good quality and can be used as is. Otherwise, additional steps to identify and eliminate potential outliers are undertaken.

The strategy used to re-estimate the tensor depends on the number of supporting triplets in the set of points. When the proportion of supporting triplets is high, this means that the quality of the tensor is not good mainly because of the presence of a few strong outliers. In this case, a statistical method based on the so-called x84 rule (Fusiello et al., 1999) is used. Absolute deviations of all triplets' residual error are calculated, from which a threshold is set as the 5.2 MAD (Median Absolute Deviation). Points having larger deviations are considered outliers and are eliminated.

In the opposite situation, i.e. when the number of supporting triplets is relatively low, then the current tensor is not able to guide the identification of outliers. Cross-correlation has to be performed on each

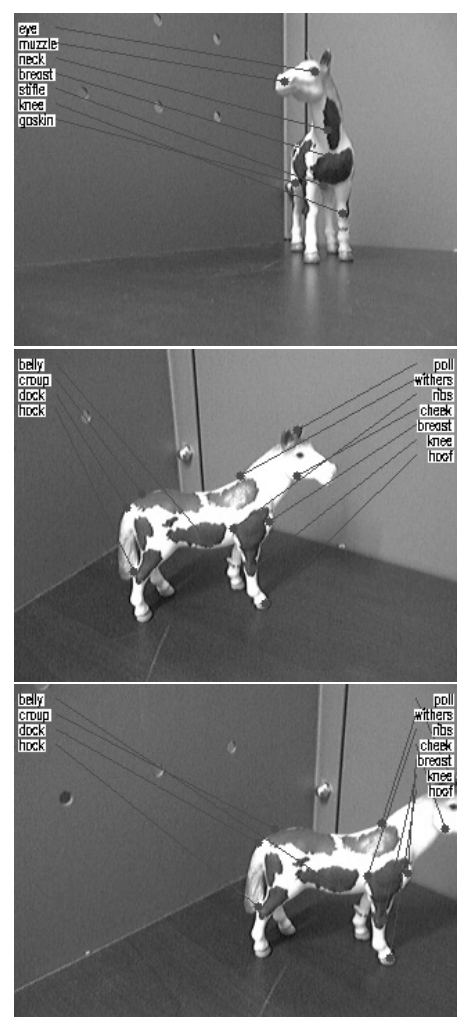

Figure 4: Few frames of an online label insertion sequence using the reference images of Figure 5.

putative triplet. All features on the current frame that do not correlate well their potential correspondences on both reference frames are rejected.

Once the outliers are rejected using one or the other of these methods, the tensor has to be re-estimated with all remaining triplets and its quality needs again to be re-evaluated. The validity of the proposed estimation scheme is illustrated in Figure 3. The accuracy of the estimated tensor, in terms of transfer error (in pixels), has been evaluated for each frame of a long sequence where the camera was freely moved around the scene of interest. The first graph is the result of tensor estimation using only the Algebraic method. The obtained errors illustrate well the necessity of using additional steps to refine the tensor estimates. The second graph shows how the introduction of the fixed-projection-matrices constraint stabilizes the results. Finally, the extra robust estimation steps further improve the results by eliminating the remaining outliers in the match set as shown in the bottom graph.

\section{RESULTS}

Figures 4 to 7 presents few images showing results obtained when running our scene augmenta- 
tion application. The system runs at approximately 14 frames/second on a regular $\mathrm{P} 41.2 \mathrm{GHz}$ computer equipped with a web cam with a resolution of $320 \times 240$

The first image of Figure 7 corresponds to the situation where a successful match has been obtained; in this case, the camera frame has been matched to the third reference image (shown in Figure 6). All the feature points that have been used to assess the validity of this match are shown in light gray (the short lines associated with each point correspond to the displacement between the two matched images). These points are also used to compute the tensor relation between that current view and two reference images (here we used reference images 1 and 2). Labels can then be displayed and are pointing to the correct location by virtue of the tensor transfer operation. The camera is moved and new images are captured, the matched point are tracked, the tensor is updated and the labels are again transferred. Figure 7 shows other images of the sequence in which the labels are indeed always pointing at the right location.

In normal operation just the labels are shown and not the feature points used for matching. This is shown in Figure 4 and 5 where, this time, the 6 reference images of Figure 5 are used to annotate the video sequence of Figure 4.

\section{CONCLUSION}

An augmented reality system has been presented where a video sequence can be augmented with textual annotations. The augmentation is accomplished by following a 2-step process. First, each incoming frame of the captured video is matched with a set of reference images. Currently a simple, but efficient, matching scheme is used where points are correlated by comparing their respective neighborhood. The fact that each putative match is validated geometrically eliminates most false matches. However, we are currently investigating other matching strategies that would make the matching process more robust to perspective variation and changes in illumination.

The second step requires continuous estimation of the trifocal tensor relation. The fact that we have in hands a reliable set of matches associated to the reference images is key in this operation. Good tensor estimates can therefore be quickly obtained using which label transfer (from the reference views where they have been inserted to the current view) becomes possible. By tracking the points over time, the tensor estimate can be updated resulting in stable label insertion.

The main advantage associated with the use of projective entities (such as the tensor and the fundamental matrix) resides in the fact that no calibration in- formation is required. The system can then easily accommodate the use of different camera, as well as zoom changes occurring during the augmentation process. Neither 3D pose information nor metric information about the scene are required.

\section{REFERENCES}

Bell, B., Hollerer, T., and Feiner, S. (2002). An annotated situation-awareness aid for augmented reality. In Proc:UIST ACM Symp. on user interface sofware and technology, pages 213-216.

Boufama, B. and Habed, A. (2005). Registration and tracking in the context of ar. ICGST Int. Journal on Graphics Vision and Image Processing, V3.

Chia, K., Cheok, A., and Prince, S. (2002). Online 6 dof augmented reality registration from natural features. In Proc. International Symposium on Mixed and Augmented Reality(ISMAR), pages 223-230.

Fusiello, A., Trucco, E., Tommasini, T., and Roberto, V. (1999). Improving feature tracking with robust statistics. Pattern Analysis and Applications, 2:312-320.

Hartley, R. and Zisserman, A. (2000). Multiple View Geometry in Computer Vision. Cambridge University Press.

Kutulakos, K. and Vallino, J. (1998). Calibration-free augmented reality. IEEE trans. on Visualization and Computer Graphics, 4:1-20.

Li, J., Laganière, R., and Roth, G. (2004). Online estimation of trifocal tensors for augmenting live video. In IEEE/ACM Symp. on Mixed and Augmented Reality, pages $182-190$.

Lourakis, M. and Argyos, A. (2004). Vision-based camera motion recovery for augmented reality. In Computer Graphics Int. Conference, pages 569-576.

Newman, J., Ingram, D., and Hopper, A. (2001). Augmented reality in a wide area sentient environmemt In Int. Symp. on Augmented Reality, pages 77-86.

Roth, G. and Whitehead, A. (2000). Using projective vision to find camera positions in an image sequence. In Proc. of Vision Interface, pages 225-232.

Vacchetti, L., Lepetit, V., and Fua, P. (2004). Stable realtime $3 \mathrm{~d}$ tracking using online and offline information. IEEE trans. on Pattern Analysis and Machine Intelligence, 26:1385-1391.

Vincent, E. and Laganière, R. (2001). Matching feature points in stereo pairs: A comparative study of some matching strategies. Machine Graphics and Vision, 10:237-259.

Vincent, E. and Laganière, R. (2002). Matching feature points for telerobotics. In IEEE Int. Workshop on Haptic Virtual Env. and Applications, pages 13-18. 

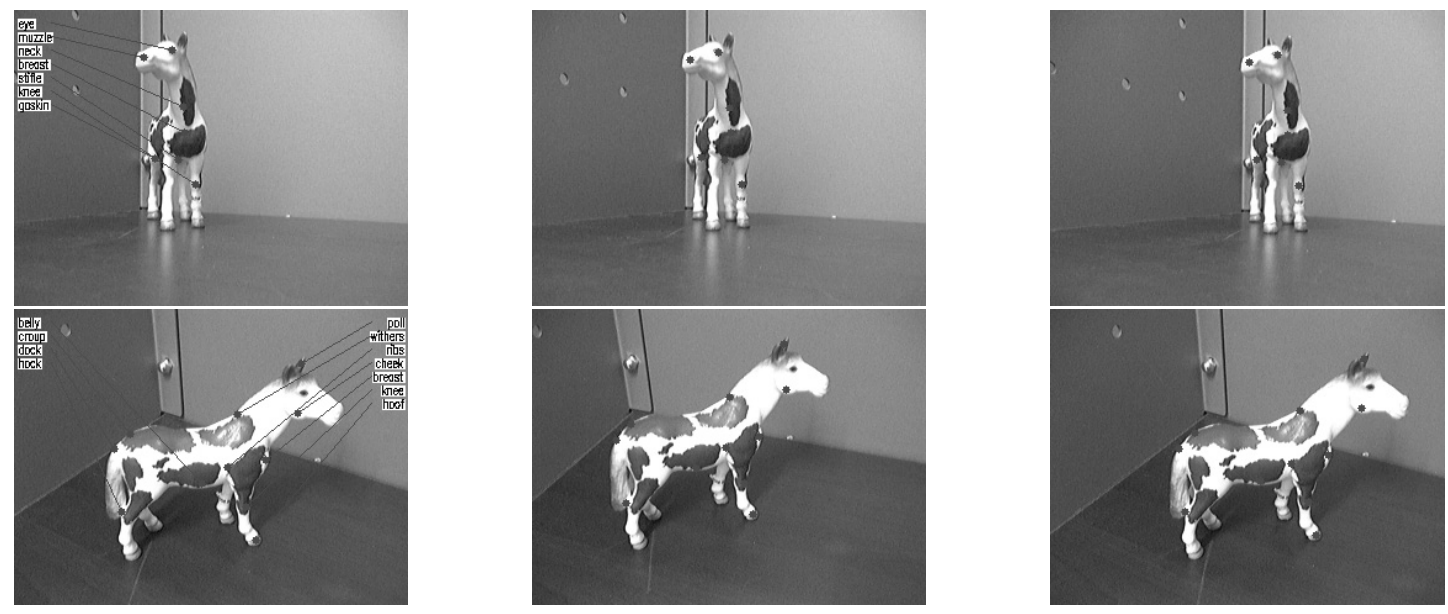

Figure 5: Annotation on the horse object. In order to accommodate changes in point of view, two series of reference images are used here. The labels have been inserted in the first image of each series, as shown here.
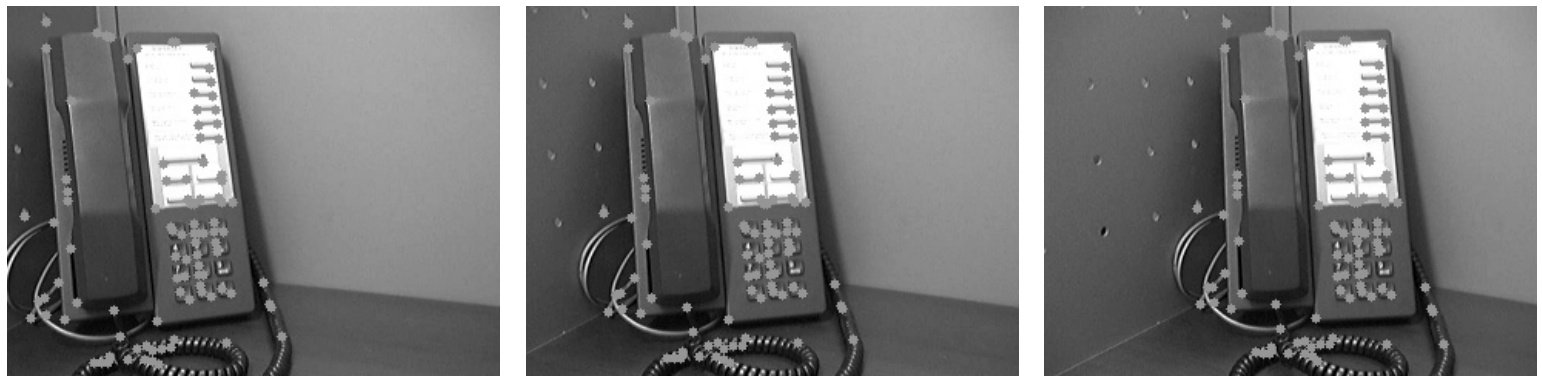

Figure 6: The three reference views and the set of matches.
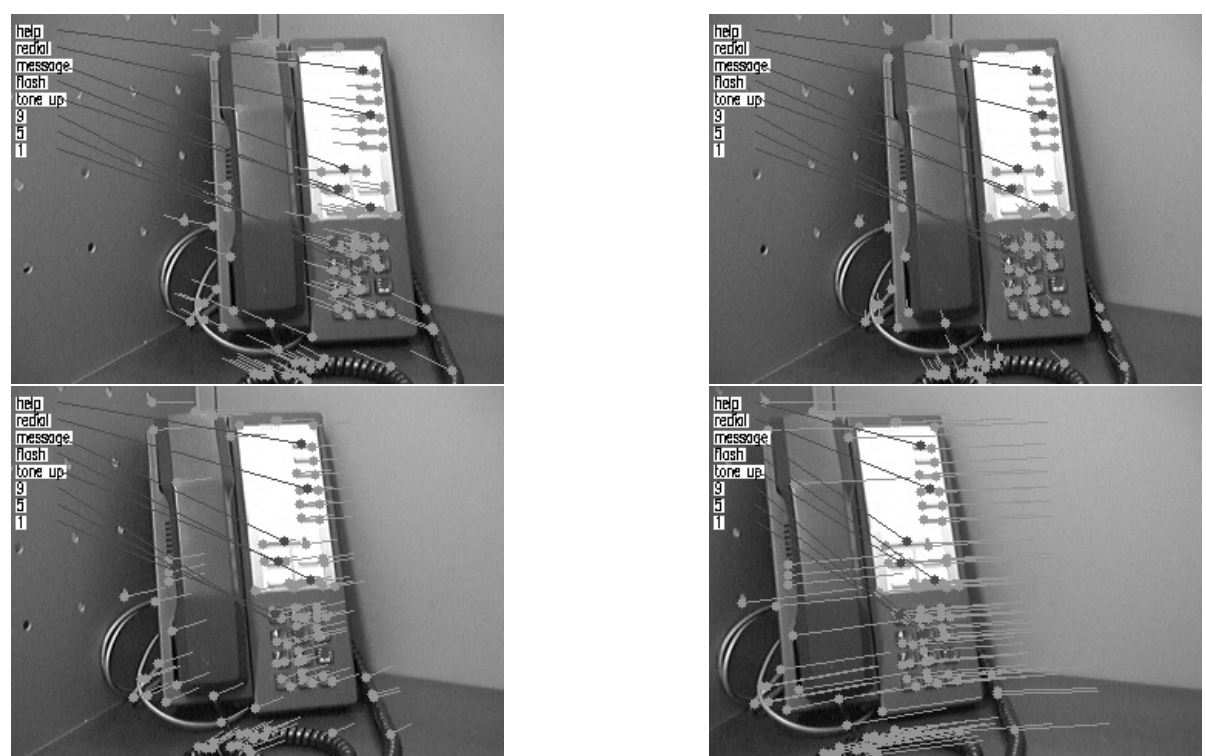

Figure 7: An image is matched with the third reference image of Figure 3, making label insertion possible; label locations are then correctly tracked over time. 